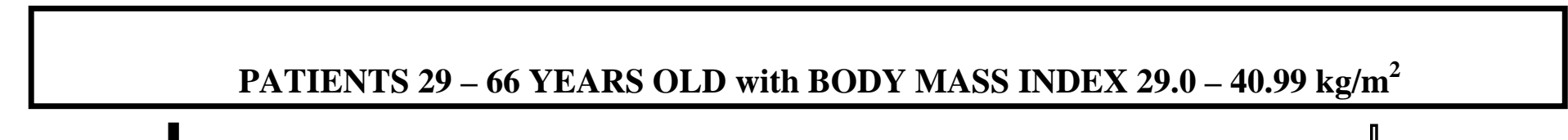

\section{INTERVENTION GROUP}

LOW FRUCTOSE DIET (LFD)

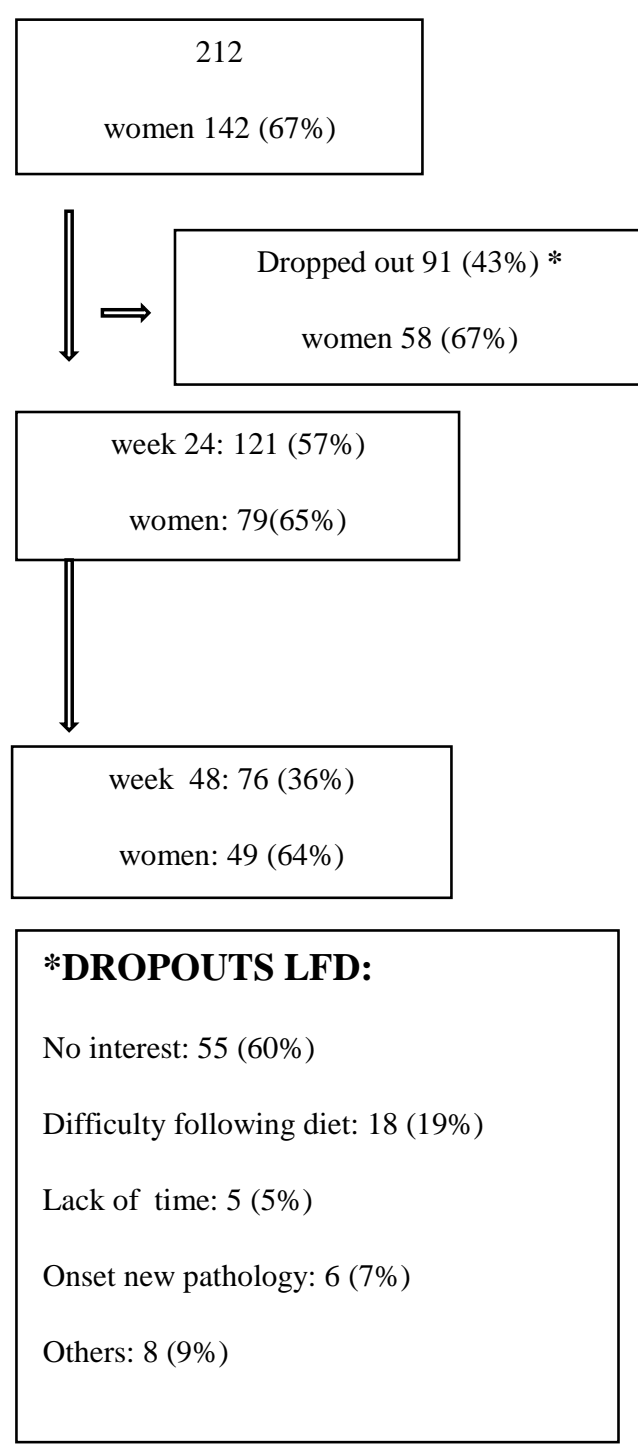

CONTROL GROUP

STANDARD DIET (SD)

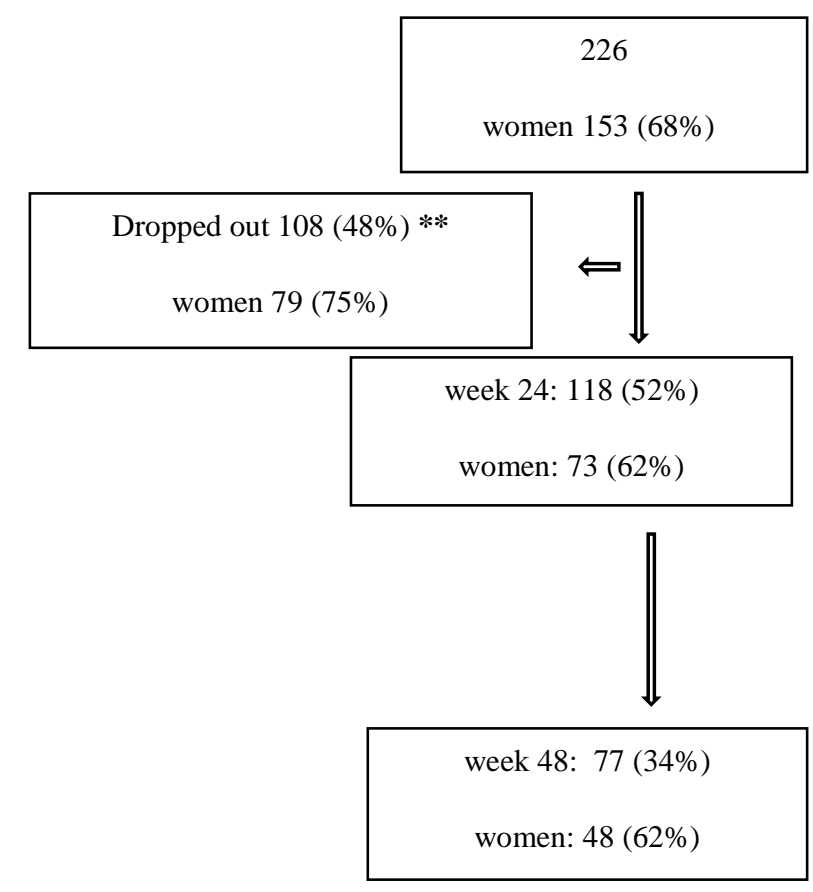

\begin{tabular}{|c|c|}
\hline & **DROPOUTS SD: \\
\hline \multirow{6}{*}{ Page 1 of 1} & No interest: 67 (62\%) \\
\hline & Difficulty following diet: 8 (7\%) \\
\hline & Lack of time: 6 (6\%) \\
\hline & Onset new pathology: 7 (6\%) \\
\hline & Physician or nurse left: 5 (5\%) \\
\hline & Others: 15 (14\%) \\
\hline
\end{tabular}




\section{Baseline characteristics}

\begin{tabular}{|c|c|c|c|c|c|c|}
\hline & \multicolumn{3}{|c|}{ Began the study* } & \multicolumn{3}{|c|}{ Completed week $24^{* *}$} \\
\hline & All (438) & $\begin{array}{l}\text { Low fructose } \\
\text { Diet (212) }\end{array}$ & $\begin{array}{l}\text { Standard diet } \\
\text { (226) }\end{array}$ & All (239) & $\begin{array}{l}\text { Low fructose } \\
\operatorname{diet}(121)\end{array}$ & $\begin{array}{l}\text { Standard diet } \\
\text { (118) }\end{array}$ \\
\hline Age (years) & $47.2 \pm 8.6$ & $46.3 \pm 8.4$ & $48.0 \pm 8.7$ & $47.9 \pm 8.6$ & $47.5 \pm 8.0$ & $48.2 \pm 9.1$ \\
\hline $\begin{array}{l}\text { Gender } \\
\text { (women) (\%) }\end{array}$ & 293 (66.9) & $141(66.5)$ & $152(67.3)$ & $152(63.6)$ & 79 (61.9) & $73(65.3)$ \\
\hline Height (cm) & $164.9 \pm 9.5$ & $165.1 \pm 9.5$ & $164.8 \pm 9.5$ & $164.5 \pm 9.9$ & $164.8 \pm 10.2$ & $164.3 \pm 9.5$ \\
\hline Weight (kg) & $94.4 \pm 13.5$ & $95.0 \pm 13.6$ & $93.9 \pm 13.4$ & $92.9 \pm 13.1$ & $92.8 \pm 12.7$ & $93.1 \pm 13.6$ \\
\hline BMI & $34.6 \pm 2.9$ & $34.8 \pm 3.0$ & $34.4 \pm 2.8$ & $34.2 \pm 2.8$ & $34.3 \pm 2.8$ & $34.2 \pm 2.7$ \\
\hline $\begin{array}{l}\text { Waist } \\
\text { circumference } \\
(\mathrm{cm})\end{array}$ & $108.2 \pm 9.2$ & $108.6 \pm 9.1$ & $107.7 \pm 9.3$ & $107.7 \pm 8.9$ & $107.9 \pm 8.7$ & $107.7 \pm 9.3$ \\
\hline $\begin{array}{l}\text { Waist } \\
\text { circumference/h } \\
\text { eight ratio }\end{array}$ & $0.656 \pm 0.050$ & $0.659 \pm 0.051$ & $0.654 \pm 0.050$ & $0.656 \pm 0.050$ & $0.657 \pm 0.050$ & $0.654 \pm 0.050$ \\
\hline $\begin{array}{l}\text { Hypertension } \\
(\%)\end{array}$ & $163(37.2)$ & $75(35.4)$ & $88(38.9)$ & $90(37.7)$ & $45(37.2)$ & $45(38.1)$ \\
\hline Hypercholestero & $180(41.1)$ & $95(42)$ & $85(40.1)$ & 105 (43.9) & $52(43.0)$ & $53(44.9)$ \\
\hline
\end{tabular}

Page 2 of 11 


\begin{tabular}{|c|c|c|c|c|c|c|}
\hline lemia (\%) & & & & & & \\
\hline $\begin{array}{l}\text { Familial } \\
\text { antecedent of } \\
\text { diabetes (\%) }\end{array}$ & $222(51.3)$ & $116(54.7)$ & $106(48)$ & 136 (56.9) & $68(56.2)$ & $68(57.6)$ \\
\hline $\begin{array}{l}\text { Smoking status } \\
(\%)\end{array}$ & & & & & & \\
\hline $\begin{array}{l}\text { - Current } \\
\text { smoker }\end{array}$ & 52 (11.9) & $18(8.5)$ & 34 (15.0) & $23(9.6)$ & $3(2.5)$ & 20 (16.9) \\
\hline $\begin{array}{l}\text { - Former } \\
\text { smoker }\end{array}$ & $93(21.2)$ & $47(22.2)$ & $46(20.4)$ & 50 (20.9) & $28(23.1)$ & 22 (18.6) \\
\hline $\begin{array}{l}\text { - Never } \\
\text { smoked }\end{array}$ & 293 (66.9) & 147 (69.3) & 146 (64.6) & 166 (69.5) & 90 (74.4) & 76 (64.4) \\
\hline $\begin{array}{l}\text { Marital status } \\
(\%)\end{array}$ & & & & & & \\
\hline $\begin{array}{l}\text {-Married or } \\
\text { cohabitating }\end{array}$ & 339 (77.4) & 166 (78.3) & 173 (76.5) & 189 (79.1) & 96 (79.3) & 93 (78.8) \\
\hline $\begin{array}{l}\text {-Divorced/ } \\
\text { separated }\end{array}$ & $28(6.4)$ & 15 (7.1) & $13(5.8)$ & $13(5.4)$ & $10(8.3)$ & 3 (2.5) \\
\hline -Widowed & $13(3.0)$ & $7(3.3)$ & $6(2.6)$ & $9(3.8)$ & $6(5)$ & 3 (2.5) \\
\hline -Single & $58(13.2)$ & 24 (11.3) & 34 (15.1) & 28 (11.7) & $9(7.4)$ & 19 (16.1) \\
\hline
\end{tabular}

Page 3 of 11 


\begin{tabular}{|c|c|c|c|c|c|c|}
\hline \multicolumn{7}{|l|}{ Social class (\%) } \\
\hline -Low & 152 (34.7) & 78 (36.8) & $74(32.7)$ & 95 (39.7) & $50(38.8)$ & $45(42)$ \\
\hline -Medium & 153 (34.9) & 78 (36.8) & 75 (33.2) & 76 (31.8) & 37 (31.1) & 39 (33.6) \\
\hline -High & 126 (28.8) & 54 (25.5) & 72 (31.9) & 64 (26.8) & 32 (29.6) & 32 (27.6) \\
\hline $\begin{array}{l}\text {-Information } \\
\text { not provided }\end{array}$ & $7(1.6)$ & $2(0.9)$ & $5(2.2)$ & $4(1.7)$ & $2(1.5)$ & $2(1.6)$ \\
\hline \multicolumn{7}{|l|}{$\begin{array}{l}\text { Blood pressure } \\
\mathrm{mmHg}\end{array}$} \\
\hline - Systolic & $128.3 \pm 15.7$ & $129.1 \pm 15.2$ & $127.6 \pm 16.1$ & $130.2 \pm 15.6$ & $130.1 \pm 15.9$ & $130.3 \pm 15.3$ \\
\hline - Diastolic & $81.4 \pm 9.8$ & $82.4 \pm 10.4$ & $80.5 \pm 9.1$ & $82.2 \pm 9.8$ & $82.6 \pm 10.7$ & $81.7 \pm 8.6$ \\
\hline $\begin{array}{l}\text { Fasting glucose } \\
\mathrm{mg} / \mathrm{dL}\end{array}$ & $91.6 \pm 12.1$ & $89.0 \pm 11.6$ & $94.0 \pm 12.1$ & $92.1 \pm 11.9$ & $89.8 \pm 11.5$ & $94.4 \pm 11.9$ \\
\hline $\begin{array}{l}\text { Fasting insulin } \\
(\mu \mathrm{U} / \mathrm{ml})\end{array}$ & $13.5 \pm 8.9$ & $13.6 \pm 10.1$ & $13.4 \pm 7.7$ & $12.8 \pm 7.1$ & $12.5 \pm 5.9$ & $13.3 \pm 8.1$ \\
\hline HOMA-2IR & $0.29 \pm 0.20$ & $0.29 \pm 0.21$ & $0.29 \pm 0.18$ & $0.28 \pm 0.16$ & $0.27 \pm 0.13$ & $0.29 \pm 0.19$ \\
\hline $\begin{array}{l}75 \text { gr OGTT } \\
\text { glucose mg/dL }\end{array}$ & $114.2 \pm 37.4$ & $111.3 \pm 37.9$ & $117.0 \pm 37.2$ & $116.2 \pm 41.5$ & $115.1 \pm 43.2$ & $118.6 \pm 39.7$ \\
\hline 75 gr OGTT & $87.0 \pm 72.7$ & $83.0 \pm 67.2$ & $90.9 \pm 77.5$ & $90.8 \pm 76.7$ & $85.1 \pm 71.5$ & $96.7 \pm 81.7$ \\
\hline
\end{tabular}

Page 4 of $\mathbf{1 1}$ 


\begin{tabular}{|l|l|l|l|l|l|l|}
\hline insulin $(\mu \mathrm{U} / \mathrm{ml})$ & & & & & & \\
\hline $\begin{array}{l}\text { Cholesterol } \\
\mathrm{mg} / \mathrm{dL}\end{array}$ & & & & & & \\
\hline- Total & $192.3 \pm 35.6$ & $192.7 \pm 35.0$ & $192.0 \pm 36.3$ & $189.9 \pm 34.9$ & $189.6 \pm 32.4$ & $189.9 \pm 37.9$ \\
\hline- LDL & $115.7 \pm 30.0$ & $116.8 \pm 31.0$ & $114.7 \pm 29.2$ & $113.6 \pm 29.6$ & $112.0 \pm 29.5$ & $114.9 \pm 30.3$ \\
\hline- HDL & $48.5 \pm 11.9$ & $48.1 \pm 11.4$ & $49.0 \pm 12.4$ & $48.5 \pm 10.9$ & $49.3 \pm 11.2$ & $47.6 \pm 10.6$ \\
\hline $\begin{array}{l}\text { Triglycerides } \\
\text { mg/dL }\end{array}$ & $140.2 \pm 75.9$ & $138.2 \pm 72.4$ & $142.1 \pm 79.1$ & $140.5 \pm 72.8$ & $139.6 \pm 72.4$ & $141.5 \pm 73.8$ \\
\hline $\begin{array}{l}\text { Physical activity } \\
\text { kcal/day }\end{array}$ & $353.8 \pm 32.2$ & $342.7 \pm 589.0$ & $364.2 \pm 671.4$ & $375 \pm 748$ & $368.1 \pm 721.2$ & $383.1 \pm 777.1$ \\
\hline- Previous 6 \\
months
\end{tabular}

Quantitative variables shown as mean \pm standard deviation.

* Between Low fructose and Standard diet in 'Began the study': Age, $\mathrm{p}=0.034$. Glucose, $\mathrm{p}<0.001$, Diastolic blood pressure, $\mathrm{p}=0.041$.

** Between Low fructose and Standard diets in 'completed week 24': Glucose, $\mathrm{p}=0.002$. Smoking status, $\mathrm{p}=0.001$. Marital status, $\mathrm{p}=0.034$ (Comparing married or cohabitant vs all other categories, $\mathrm{p}=0.53$ ). 
Nutritional values at the start of the study for those who completed week 24.

\begin{tabular}{|l|l|l|}
\hline & Low fructose diet $(\mathrm{n}=121)$ & Standard diet $(\mathrm{n}=118)$ \\
\hline kcal/day & $1900.5 \pm 515.3$ & $1841.3 \pm 518.2$ \\
\hline Proteins & $173.1 \pm 38.9$ & $177.4 \pm 35.1$ \\
\hline Fats & $344.9 \pm 80.3$ & $338.7 \pm 76.7$ \\
\hline SFA & $97.9 \pm 23.7$ & $98.00 \pm 28.9$ \\
\hline MUFA & $132.3 \pm 32.1$ & $126.9 \pm 31.1$ \\
\hline PUFA & $48.1 \pm 17.2$ & $48.3 \pm 17.7$ \\
\hline Carbohydrates & $498.2 \pm 67.6$ & $491.9 \pm 75.4$ \\
\hline Starch & $254.8 \pm 58.4$ & $238.8 \pm 65.8$ \\
\hline Lactose & $35.1 \pm 19.8$ & $39.0 \pm 25.8$ \\
\hline Total galactose & $20.1 \pm 10.8$ & $22.0 \pm 12.7$ \\
\hline Free galactose & $1.9 \pm 1.8$ & $1.7 \pm 1.6$ \\
\hline Sucrose & $111.4 \pm 42.2$ & $109.7 \pm 44.8$ \\
\hline Sucrose in natural foods & $25.2 \pm 16.0$ & $27.7 \pm 17.9$ \\
\hline $\begin{array}{l}\text { Sucrose in industrial } \\
\text { foods }\end{array}$ & $86.2 \pm 44.1$ & $82.0 \pm 45.8$ \\
\hline Total fructose & $102.5 \pm 32.8$ & $104.1 \pm 41.3$ \\
\hline Free fructose & $47.6 \pm 29.1$ & $50.2 \pm 34.9$ \\
\hline $\begin{array}{l}\text { Total fructose in natural } \\
\text { foods }\end{array}$ & $45.4 \pm 33.6$ & $52.2 \pm 41.3$ \\
\hline $\begin{array}{l}\text { Total fructose in } \\
\text { industrial foods }\end{array}$ & $57.1 \pm 26.7$ & $51.9 \pm 29.4$ \\
\hline $\begin{array}{l}\text { Free fructose in natural } \\
\text { foods }\end{array}$ & $33.1 \pm 28.4$ & $38.4 \pm 36.1$ \\
\hline
\end{tabular}

Page 6 of 11 


\begin{tabular}{|l|l|l|}
\hline $\begin{array}{l}\text { Free fructose in } \\
\text { industrial foods }\end{array}$ & $14.5 \pm 14.1$ & $11.8 \pm 15.4$ \\
\hline Total glucose & $90.0 \pm 27.3$ & $89.3 \pm 30.9$ \\
\hline Free glucose & $38.3 \pm 20.7$ & $37.6 \pm 20.3$ \\
\hline $\begin{array}{l}\text { Total glucose in natural } \\
\text { foods }\end{array}$ & $32.6 \pm 23.3$ & $36.2 \pm 24.1$ \\
\hline $\begin{array}{l}\text { Free glucose in natural } \\
\text { foods }\end{array}$ & $23.4 \pm 19.6$ & $25.7 \pm 19.4$ \\
\hline $\begin{array}{l}\text { Total glucose in } \\
\text { industrial foods }\end{array}$ & $57.3 \pm 25.2$ & $53.1 \pm 27.0$ \\
\hline $\begin{array}{l}\text { Free glucose in } \\
\text { industrial foods }\end{array}$ & $14.9 \pm 12.2$ & $12.0 \pm 11.9$ \\
\hline Fiber* & $11.1 \pm 6.5$ & $10.6 \pm 3.4$ \\
\hline
\end{tabular}

Mean nutritional density of daily intakes \pm SD. *Grams per 1000 kcal.

SFA: saturated fatty acids; MUFA: monounsaturated fatty acids; PUFA: polyunsaturated fatty acids. 
Primary and secondary outcomes. Week 24-week 0.

\begin{tabular}{|c|c|c|c|c|c|c|c|}
\hline & \multicolumn{3}{|c|}{ Low fructose diet $(n=121)$} & \multicolumn{3}{|c|}{ Standard diet $(\mathrm{n}=118)$} & \multirow{2}{*}{$\begin{array}{l}\text { Differences } \\
\text { between } \\
\text { diets (LFD- } \\
\text { SD) }\end{array}$} \\
\hline & Week 0 & Week 24 & $\begin{array}{l}\text { Difference } \\
(24-0)\end{array}$ & Week 0 & Week 24 & $\begin{array}{l}\text { Difference } \\
(24-0)\end{array}$ & \\
\hline $\begin{array}{l}\text { Fasting glucose } \\
(\mathrm{mg} / \mathrm{dL})\end{array}$ & $89.8 \pm 11.5$ & $85.2 \pm 10.9$ & -4.6 & $94.4 \pm 11.9$ & $92.4 \pm 11.5$ & -2.0 & -2.7 \\
\hline $\begin{array}{l}\text { Fasting insulin } \\
(\mu \mathrm{U} / \mathrm{mL})\end{array}$ & $12.5 \pm 5.9$ & $11.0 \pm 5.8$ & -1.6 & $13.3 \pm 8.1$ & $11.7 \pm 7.4$ & -1.6 & 0.0 \\
\hline HOMA-2IR & $\begin{array}{l}0.2727 \\
\pm 0.1297\end{array}$ & $\begin{array}{l}0.2306 \\
\pm 0.1296\end{array}$ & -0.0421 & $\begin{array}{l}0.2966 \\
\pm 0.1920 \\
\end{array}$ & $\begin{array}{l}0.2590 \\
\pm 0.1692 \\
\end{array}$ & -0.0376 & -0.0045 \\
\hline BMI & $34.3 \pm 2.8$ & $31.9 \pm 3.3$ & -2.4 & $34.2 \pm 2.7$ & $32.2 \pm 3.0$ & -2.0 & -0.400 \\
\hline $\begin{array}{l}\text { Waist circumference } \\
\text { (cm) }\end{array}$ & $107.9 \pm 8.7$ & $100.9 \pm 10.3$ & -7.0 & $107.7 \pm 9.3$ & $102.0 \pm 11.8$ & -5.7 & -1.304 \\
\hline $\begin{array}{l}\text { Waist } \\
\text { circumference/height } \\
\text { ratio }\end{array}$ & $0.66 \pm 0.05$ & $0.62 \pm 0.06$ & -0.04 & $0.65 \pm 0.05$ & $0.62 \pm 0.07$ & -0.03 & -0.01 \\
\hline Weight (kg) & $92.8 \pm 12.7$ & $86.3 \pm 13.5$ & -6.5 & $93.1 \pm 13.6$ & $87.6 \pm 13.3$ & -5.5 & -1.0 \\
\hline $\begin{array}{l}\text { Fasting total cholesterol } \\
\text { (mg/dL) }\end{array}$ & $189.6 \pm 32.4$ & $187.6 \pm 32.2$ & -1.9 & $189.9 \pm 37.9$ & $187.1 \pm 34.7$ & -2.9 & 0.9 \\
\hline Fasting HDL (mg/dL) & $49.3 \pm 11.2$ & $50.3 \pm 11.4$ & 1.0 & $47.6 \pm 10.6$ & $48.7 \pm 11.8$ & 1.1 & 0.1 \\
\hline Fasting LDL (mg/dL) & $112.0 \pm 29.5$ & $112.3 \pm 29.4$ & 0.3 & $114.9 \pm 30.3$ & $112.4 \pm 28.3$ & -2.5 & 2.8 \\
\hline $\begin{array}{l}\text { Fasting Triglycerides } \\
(\mathrm{mg} / \mathrm{dL})\end{array}$ & $139.6 \pm 72.4$ & $128.4 \pm 73.9$ & -11.1 & $141.5 \pm 73.8$ & $129.6 \pm 70.1$ & -11.9 & 0.8 \\
\hline $\begin{array}{l}\text { Sistolic blood pressure } \\
\text { (mmHg) }\end{array}$ & $130.1 \pm 15.9$ & $124.5 \pm 13.6$ & -5.6 & $130.3 \pm 15.3$ & $126.1 \pm 13.9$ & -4.1 & -1.5 \\
\hline $\begin{array}{l}\text { Diastolic blood pressure } \\
\text { (mmHg) }\end{array}$ & $82.6 \pm 10.7$ & $80.4 \pm 9.1$ & -2.2 & $81.7 \pm 8.6$ & $79.2 \pm 9.4$ & -2.5 & 0.3 \\
\hline
\end{tabular}


Mean \pm SD

Page $\mathbf{9}$ of $\mathbf{1 1}$ 
Primary and secondary outcomes. Week 48-week 24.

\begin{tabular}{|c|c|c|c|c|c|c|c|}
\hline & \multicolumn{3}{|c|}{ Low fructose diet $(n=76)$} & \multicolumn{3}{|c|}{ Standard diet $(\mathrm{n}=77)$} & \multirow{2}{*}{$\begin{array}{l}\text { Differences } \\
\text { between diets } \\
\text { (LFD-SD) }\end{array}$} \\
\hline & Week 24 & Week 48 & $\begin{array}{l}\text { Difference } \\
(48-24)\end{array}$ & Week 24 & Week 48 & $\begin{array}{l}\text { Difference } \\
(48-24)\end{array}$ & \\
\hline $\begin{array}{l}\text { Fasting glucose } \\
(\mathrm{mg} / \mathrm{dL})\end{array}$ & $85.08 \pm 9.2$ & $93.2 \pm 10.1$ & 8.1 & $92.8 \pm 12.3$ & $94.3 \pm 11.2$ & 1.4 & 6.7 \\
\hline $\begin{array}{l}\text { Fasting insulin } \\
(\mu \mathrm{U} / \mathrm{mL})\end{array}$ & $11.2 \pm 6.1$ & $10.3 \pm 4.8$ & -0.9 & $11.3 \pm 7.9$ & $13.0 \pm 10.1$ & 1.7 & -2.6 \\
\hline HOMA-2IR & $0.234 \pm 0136$ & $0.228 \pm 0.104$ & -0.006 & $0.251 \pm 0.178$ & $0.288 \pm 0.231$ & 0.038 & -0.044 \\
\hline BMI & $31.9 \pm 3.2$ & $32.1 \pm 3.1$ & 0.2 & $32.4 \pm 3.1$ & $32.0 \pm 3.4$ & 0.6 & -0.4 \\
\hline $\begin{array}{l}\text { Waist circumference } \\
(\mathrm{cm})\end{array}$ & $100.3 \pm 10.1$ & $100.3 \pm 9.5$ & 0.0 & $103.2 \pm 9.4$ & $104.3 \pm 9.0$ & 1.16 & -1.16 \\
\hline $\begin{array}{l}\text { Waist } \\
\text { circumference/height } \\
\text { ratio }\end{array}$ & $0.614 \pm 0.057$ & $0.615 \pm 0.053$ & 0.001 & $0.626 \pm 0.052$ & $0.633 \pm 0.052$ & 0.007 & -0.006 \\
\hline Weight (kg) & $85.8 \pm 13.4$ & $86.2 \pm 12.8$ & 0.4 & $88.4 \pm 13.5$ & $89.9 \pm 14.1$ & 1.5 & -1.1 \\
\hline $\begin{array}{l}\text { Fasting total cholesterol } \\
(\mathrm{mg} / \mathrm{dL})\end{array}$ & $187.8 \pm 33.3$ & $199.8 \pm 34.0$ & 12.0 & $184.8 \pm 32.2$ & $194.5 \pm 36.5$ & 10.2 & 2.2 \\
\hline Fasting HDL (mg/dL) & $49.6 \pm 11.7$ & $52.5 \pm 13.0$ & 3.1 & $48.4 \pm 11.6$ & $50.9 \pm 12.9$ & 2.4 & 0.7 \\
\hline Fasting LDL (mg/dL) & $111.5 \pm 30.6$ & $119.1 \pm 31.6$ & 7.6 & $110.7 \pm 22.3$ & $116.7 \pm 31.8$ & 6.0 & 1.6 \\
\hline $\begin{array}{l}\text { Fasting Triglycerides } \\
\text { (mg/dL) }\end{array}$ & $137.0 \pm 83.4$ & $130.7 \pm 70.6$ & -6.3 & $126.1 \pm 59.6$ & $131.5 \pm 78.0$ & 5.4 & -11.7 \\
\hline $\begin{array}{l}\text { Sistolic blood pressure } \\
\text { (mmHg) }\end{array}$ & $124.0 \pm 14.2$ & $123.0 \pm 14.1$ & -1 & $125.2 \pm 14.7$ & $126.3 \pm 14.7$ & 1.1 & -2.1 \\
\hline $\begin{array}{l}\text { Diastolic blood pressure } \\
\text { (mmHg) }\end{array}$ & $80.3 \pm 9.9$ & $77.8 \pm 9.5$ & -2.5 & $79.1 \pm 9.3$ & $81.2 \pm 13.4$ & 2.1 & -4.6 \\
\hline
\end{tabular}

Mean \pm SD 


\section{$\underline{\text { Adverse events }}$}

Only 5 adverse events were observed, all in the Low fructose diet group: 3 participants reported constipation, 1 hypotension, and 1 general weakness. All events were transitory, and all 5 participants completed the study. No adverse events were reported in the Standard diet group. 